\title{
Will the Modern Technology ever Catch up with the 'God's Formula' of Dietary Fiber?
}

\author{
Shigeyuki Nakaji* \\ Department of Social Medicine, Hirosaki University Graduate School of Medicine, Japan
}

*Corresponding author: Shigeyuki Nakaji, Department of Social Medicine, Hirosaki University Graduate School of Medicine, 5 Zaifucho, 036-8562 Hirosaki City, Aomori, Japan.

To Cite This Article: Shigeyuki Nakaji, Will the Modern Technology ever Catch up with the 'God's Formula' of Dietary Fiber?. Am J Biomed Sci \& Res. 2020 - 7(2). AJBSR.MS.ID.001133. DOI: 10.34297/AJBSR.2020.07.001133.

Received: 眥 January 31, 2020; Published: 眥 February 06, 2020

\section{Opinion}

The history of dietary fiber began back in ancient Europe during Hippocratic era, where function of wheat bran was already known as a laxative. The development of powdering system in France during $18^{\text {th }}$ century has led to a debate between white bread and brown bread. However, this debate was based solely on their nutritional values, and depended on the opinions of food manufacturing companies and certain dietitians, thus the significance of dietary fiber was not well explored.

In the USA, Kellogg et al. [1] had found a great interest in the function of wheat bran and advanced the scientific research on it. During the process, Williams et al. [2] have found that although cellulose, hemicellulose and lignin are degraded in digestive tracts, they increase the amount of stool and softens it. Also, McCarrison [3] concluded from the research that eating whole-wheat bread, beans and fruits are beneficial for human health. However, the debate on dietary fiber was still depended on the opinions of food manufacturing companies and certain dietitians.

In terms of the relationship between dietary fiber and chronic lifestyle disease, Walker [4] conducted a research on Bantu tribes in South Africa in 1940s, and suggested that low prevalence of heart disease and atherosclerosis among the tribe was due to their lowfat and high-fiber dietary habit. Cleave [5] claimed that high consumption of refined carbohydrate food, especially sugar and white flour led to obesity, diabetes, colon diseases etc., which are also known as the Saccharin disease. The interest of dietary fiber then finally caught attention of medical doctors and researchers.

In 1953, dietary fiber was originally defined by Hipsley [6], and it included the definition of cellulose, hemicellulose and lignin as well.

In 1960s, Trowel [7] reported that the reasons why certain diseases are common in western Europe and not in Africa (constipa tion, diverticulosis, colorectal cancer, ulcerative colitis etc.) are due to the amount of dietary fiber intake in their diet.

In 1971, Burkitt [8] introduced the dietary fiber hypothesis, where he stated a significant association between dietary fiber and colorectal cancer. As a result, more researches on dietary fiber and diseases associated with large bowel (cancer, diverticulosis, constipation etc....) were conducted. Also, it led to more research not only on diseases related to large bowel, but also other diseases such as diabetes mellitus, obesity and ischemic heart disease as well. The newly discovered function of dietary fiber other than that on large bowel function, including inhibition of calorie intake and preventive effect of absorption, had a large impact on the researchers.

The dietary fiber hypothesis was supported for many years, however, Fuchs et al. [9] published the research in 1999 reporting the completely opposite results. Their cohort research included administering food-related surveys to 88,757 females (34-59 years old) in 1980, and then followed them up for the next 16 years. During the period, 787 cases of colorectal cancer were found. As an overall result, there was no significant association between the amount of dietary fiber intake and incidence of colorectal cancer. There were several researches conducted in the USA afterwards, which resulted in supporting Fuchs' findings. There were a few researches that still supported the dietary fiber hypothesis; however, the impact of Fuchs' research was tremendous, and medical research on dietary fiber became stagnant in $21^{\text {st }}$ century.

Such trend was ceased by the increasing interests in intestinal bacteria research. Notable progression and development of method to analyze intestinal bacteria using DNA analysis have accelerated the research of intestinal bacteria, which was once known as the 'black box' of human body. Since most of the substrate for degradation and fermentation is dietary fiber, the interest of intestinal 
bacteria subsequently increased the interest for dietary fiber (prebiotics).

At the same time, many developed countries started facing ageing society, that involved increased number of elderly adults with dementia. Due to its association with dementia, mastication then gained attention as preventive factor for cognitive function decline [10]. Thus, dietary fiber that promotes mastication has been gaining attention.

Five major functions of dietary fiber that are currently known are as follows:

a. To increase stool volume and to maintain colon functions

b. To prevent obesity by reducing calorie intake

c. To prevent diabetes mellitus by delaying absorption of nutrients

d. To affect the health of whole body through intestinal bacteria

e. To prevent dementia by promoting mastication

As well as dementia, the world is now facing an increasing prevalence of overeating and subsequent obesity, diabetes mellitus, arteriosclerosis and cardiovascular diseases. The same trend was emphasized when the dietary fiber hypothesis was introduced by Burkitt. 50 years on, the amount of attention gained due to the importance of dietary fiber is greater than ever. This is since we have reached the era of food satiation, where controlling the amount of dietary intake became an important medical and societal issues.

Development of refining technology in recent years have been allowing us to consume highly nutritious food, in other words, less dietary fiber-rich food, as dietary fiber is considered non-nutritive. This is causing obesity and then to increased prevalence of related diseases. Thus, it is important to consume non-nutritive (rich in dietary fiber) food. Such concept did not exist when people were suffering from famine and shortage of food in the past. Therefore, dietary fiber is a big hope in the food satiety era.

In addition, we need to pay attention to the way dietary fiber is consumed. We often focus only on the amount of dietary fiber intake; however, the type and the contents of dietary fiber is also extremely important. There are variety of dietary fiber in foods, from soluble and non-soluble, to different types such as cellulose, hemicellulose, lignin, pectin, guar gam etc...., and they all have different functions. If dietary fiber functions when they are balanced, each dietary fiber needs to be consumed in the appropriate/balanced amount. To be able to achieve that, variety of plant-based food need to be consumed. Some plant-based food contains the dietary fiber in the perfect balance, known to have 'God's formula', although people have rapidly diverted from consuming such food in the past half century. As humans have been having eating habit that is rich in dietary fiber for centuries, we must aim to consume the same amount of dietary fiber as the past, with food rich in the right amount and balance of dietary fiber, or 'God's formula', as that's how our body have adapted and developed in the first place. Before dietary fiber gained attention, the world of dietetics was only about nutrients. Many scientists emerged in this field, producing Noble prize winners as well. When dietary fiber gained more attention, many researchers warned the excessive intake of nutrients, which created a different dimension in the world of dietetics - with nutrients being a 'light' and dietary fiber being a 'shadow' in the dietetics field.

Dietary fiber has become a big hope in dietetics field with its wide variety of functions including the effect on intestinal bacteria as well as prevention of constipation, Saccharin disease, colon diseases, obesity, diabetes mellitus and dementia. The existence of dietary fiber has changed from something that only certain dietitian cared about, to something that all the scientists as well as any ordinary people consider or think about in their everyday lives.

The future of dietary fiber should consist of an establishment of methodology that can maximize the amount of dietary fiber intake, despite its commonly unpleasant flavor and texture. Therefore, what need to be done to increase dietary fiber consumption are; for each individual to understand the importance of dietary fiber, and for food manufacturers and scientists to develop a plant-based food material as well as cooking method to significantly improve flavor and texture of fiber-rich food, and to invent a technology to produce them.

\section{References}

1. Kellogg JH (1923) The New Dietetics; a Guide to the Scientific Feeding in Health and Disease, Battle Creek.

2. Williams RD, WH Olmsted (1936) The effect of cellulose, hemicellulose and lignin on the weight of the stool; a contribution to the study of laxation in man J Nutr 11: 433.

3. Mc Carrison R (1936) Nutrition and health, cantor lectures before Royal Society of Arts. Faber and Faber, London. 29: 103-104.

4. Walker ARP, UB Arvidsson (1954) Fat intake, serum cholesterol concentration, and atherosclerosis in the South African Bantu. I. Low fat intake and the age trend of serum cholesterol concentration in the South African Bantu. J Clin Invest 33(10): 1358-1365.

5. Cleave TL (1956) The neglect of natural principles in current medical practice. J Roy Nav Med Serv 42: 54-83.

6. Hipsley EH (1953) Dietary fiber and pregnancy toxaemia. Brit Med J 2: 420-422.

7. Trowell HC (1960) Non-infective disease in Africa, Edward Arnold, London p. 217.

8. Burkitt DP (1971) Epidemiology of cancer of the colon and rectum. Cancer 28: 3-13.

9. Fuchs CS, EL Giovannucci, GA Colditz, DJ Hunter, MJ Stampfer, et al. (1999) Dietary fiber and the risk of colorectal cancer and adenoma in women. N Engl J Med 340(3): 169-176.

10. Chen H, Iinuma M, Onozuka M, Kubo KY (2015) Chewing maintains hippocampus-dependent cognitive function. Int J Med Sci 12(6): 502509. 